\title{
An AP Selection Criteria for Enhanced Indoor Positioning using IEEE 802.11 RSSI Measurements and AP Configuration Information
}

\author{
Jun Gyu Hwang*, Kwang Eog Lee** and Joon Goo Park ${ }^{\dagger}$
}

\begin{abstract}
The demand for LBS (Location Based Services) is increasing in the development of communication and mobile technologies. Positioning technology is a core technology for LBS, because LBS is based on a position of each device or user. But positioning technology especially for indoor environments is getting a lot of attention. Indoor positioning errors usually occur seriously in indoor environments where APs (Access Points) are set in a very concentrated and complex arrangement. In order to reduce indoor positioning errors, we adopt DOP (Dilution of Precision) which reflects an APs configuration information. In this paper, we propose an enhanced indoor positioning method using IEEE 802.11 RSSI measurements and AP configuration information.
\end{abstract}

Keywords: Indoor positioning, RSSI, Dilution of precision (DOP)

\section{Introduction}

With a rapid increase of mobile data services and multimedia business, the position information of mobile devices is getting more important. Especially in complex indoor environments, such as airport terminals, exhibition halls, warehouses, supermarkets, libraries, underground parkings, mine environments; reliable and accurate position information, which is more difficult to obtain, is needed for upcoming LBSs. Indoor positioning is somewhat different from outdoor positioning from the point of reference radio propagation environments. Indoor positioning environments where reference radio access points are set in a very concentrated and complex arrangement will generate a larger amount of position errors compared with that of outdoor positioning using a benign radio reference such as GPS [1]. Moreover, indoor positioning performance range is relatively short, so the demanded positioning precision is relatively higher than that of outdoor positioning.

To meet this requirement of positioning accuracy, various indoor positioning methods using IR [2], RFID [3], ultrasonic, and UWB [4] have been studied. But there are not a lot of researches to enhance the positioning accuracy by considering the geometric configuration of reference radio APs.

In this paper, we propose an enhanced indoor positioning algorithm, based on the existing indoor positioning method using IEEE 802.11 RSSI (Received Signal Strength Indicator) measurements [6,7], which considers the geometric configuration information of APs for more accurate positioning.

$\dagger$ Corresponding Author: Graduate School of Electronics Engineering, Kyungpook National University, Korea. (jgpark@knu.ac.kr)

* Graduate School of Electronics Engineering, Kyungpook National University, Korea. (cjstk891015@naver.com)

** Agency for Defence Development Daejeon, Korea. (kelee@add.re.kr)

Received: October 8, 2015; Accepted: November 26, 2015
This paper is composed of four sections. Section 1 is the Introduction section. Section 2 describes the theoretical backgrounds of RSSI and dilution of precision (DOP). Section 3 describes the relationship between positioning error with RSSI and DOP. The experimental results and conclusions are given in section 4 and 5 , respectively.

\section{Theoretical Background}

\subsection{Dilution of Precision (DOP)}

The dilution of precision reflects the geometric information of APs which are used for positioning [8].

Fig. 1 shows a scenario where a GPS receiver measures the pseudo-range of two satellites. Two geometries are indicated.

In Fig. 1, the case on the left side, the satellites are located approximately at right angles with respect to the user location. In the case on the right side, the angle between the satellites as viewed from the user is much smaller. The figure shows that the area of uncertainty on the position of the receiver is smaller than that of the case on the right. The area of uncertainty is marked by black. In other words: Satellites that appear farther apart in the sky provide a more accurate position solution than those close

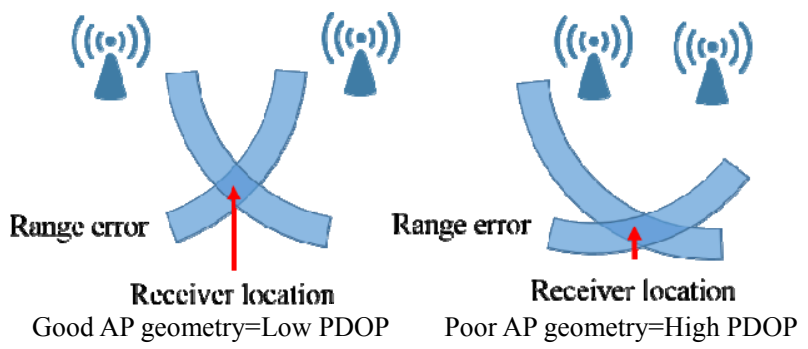

Fig. 1. Relative geometry and dilution of precision 
together. According to this idea, if we use an AP instead of a satellite, if the APs appear farther apart in indoor environments they can provide a more accurate position solution than those close together.

In this section, we will adopt DOP of APs for an indoor WLAN positioning system. This paper does not consider the vertical positioning of an object, so we just determine the two-dimensional position. As a first step in computing the DOP, we consider the unit vectors from the receiver to AP i [9]:

$$
P_{i}=\left[\frac{\left(\mathrm{x}_{\mathrm{i}}-\mathrm{x}\right)}{\mathrm{R}_{\mathrm{i}}} \frac{\left(\mathrm{y}_{\mathrm{i}}-\mathrm{y}\right)}{\mathrm{R}_{\mathrm{i}}}\right]
$$

where:

$$
\mathrm{R}_{\mathrm{i}}=\sqrt{\left(\mathrm{x}_{\mathrm{i}}-\mathrm{x}\right)^{2}+\left(\mathrm{y}_{\mathrm{i}}-\mathrm{y}\right)^{2}}
$$

$\mathrm{x}, \mathrm{y}$ : denote the coordinates of the receiver

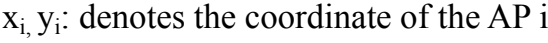

Formulate the matrix, A, as:

$$
A=\left[\begin{array}{c}
\frac{\left(x_{1}-x\right)}{R_{1}} \frac{\left(y_{1}-y\right)}{R_{1}} 1 \\
\frac{\left(x_{2}-x\right)}{R_{2}} \frac{\left(y_{2}-y\right)}{R_{2}} 1 \\
\frac{\left(x_{3}-x\right)}{R_{3}} \frac{\left(y_{3}-y\right)}{R_{3}} 1
\end{array}\right]
$$

The first two elements of each row in $\mathrm{A}$ are the components of a unit vector from the receiver to the indicated AP.

A relationship for commonly known GDOP (Geometry Dilution of Precision) is obtained in terms of the elements of $\mathrm{H}$ by expressing $\left(\mathrm{A}^{\mathrm{T}} \mathrm{A}\right)^{-1}$ in component form

$$
H=\left(A^{T} A\right)^{-1}=\left[\begin{array}{ccc}
d_{x}^{2} & d_{x y}^{2} & 1 \\
d_{x y}^{2} & d_{y}^{2} & 1 \\
d_{x z}^{2} & d_{y z}^{2} & 1
\end{array}\right]
$$

The elements of $\mathrm{H}$ are strictly covariances of geometric terms. Therefore, $d_{i j}$, as in dilution of precision, is used.

GDOP expresses the estimation error in terms of the geometry of the AP positions. It is defined as:

$$
\mathrm{GDOP}=\sqrt{\operatorname{trace}(\mathrm{H})}
$$

Using Eq. (4),

$$
\mathrm{GDOP}=\sqrt{\mathrm{d}_{\mathrm{x}}^{2}+\mathrm{d}_{\mathrm{y}}^{2}+1}
$$

The meaning of various DOP values is described in [9].

Table 1. Rating of DOP

\begin{tabular}{c|c}
\hline DOP Value & Rating \\
\hline$<1$ & Ideal \\
\hline $1-2$ & Excellent \\
\hline $2-5$ & Good \\
\hline $5-10$ & Moderate \\
\hline $10-20$ & Fair \\
\hline$>20$ & Poor \\
\hline
\end{tabular}

\subsection{Received Signal Strength Indicator (RSSI)}

Received signal strength indicator (RSSI) is the ratio of transmitter power and received power present in $\mathrm{dBm}$. The RSSI has the characteristic that it can decrease exponentially according to an increase on the distance. Because of these characteristic, we will use the RSSI attenuation model and is given as $[10,11]$ :

$$
\begin{gathered}
\operatorname{RSSI}[\mathrm{dBm}]=-\left(10 \log _{10} \mathrm{~d}-\mathrm{A}\right) \\
\text { Distance }[\mathrm{m}]=10^{\frac{\mathrm{RSSI}-\mathrm{A}}{-10 \mathrm{n}}}
\end{gathered}
$$

where:

$\mathrm{n}=$ signal propagation constant, also named propagation exponent

$\mathrm{d}=$ distance from sender to receiver

$\mathrm{A}=$ received signal strength at a distance of 1 meter

Fig. 2 shows RSSI attenuation results according to the elapsed distance.

According to Fig. 2, we can find that the attenuation log model will calculate more accurate distance information from RSSI measurements, from $1 \mathrm{~m}$ to $7 \mathrm{~m}$. And we use average RSSI for each distance when calculating the attenuation log model. The RSSI for each distance has a variance of about 2. The Relationship between Positioning Error with RSSI and DOP

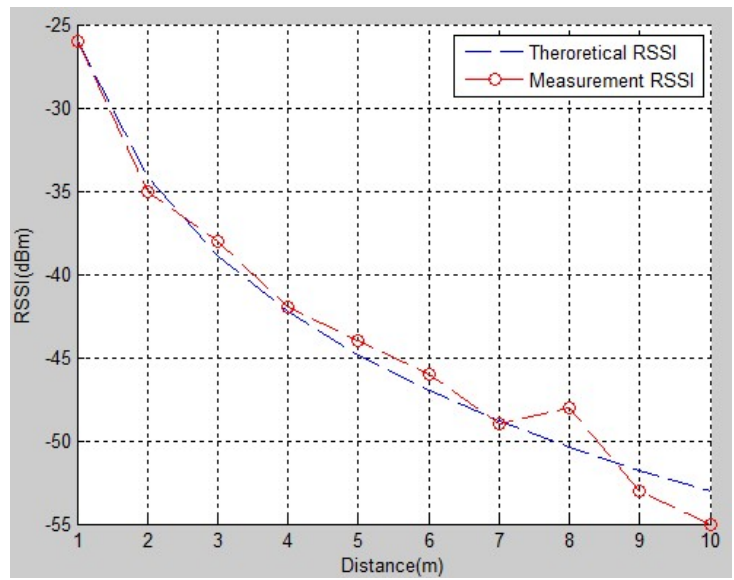

Fig. 2. RSSI attenuation according to the elapsed distance 


\section{The Relationship between Positioning Error with RSSI and DOP}

To lower indoor positioning error, we usually select the AP set which provides a strong signal strength without considering the distribution information of the corresponding APs, directly impacting on positioning errors. The low DOP value of the selected AP set means that the selected AP combination has good geometric distribution. If the AP combination has high RSSI values and low DOP value, we can expect potentially reduced positioning errors. However, it is difficult to satisfy all conditions simultaneously. In order to overcome the problem, we consider selecting the AP combination with an appropriate trade-off range between RSSI measurements and DOP values.

As shown in Fig. 4, the distance between AP1 and MS (Mobile Station) is indicated by $\mathrm{d}_{1}$, in the same way, the distances between AP2, AP3, AP4 and MS are indicated by $\mathrm{d}_{2}, \mathrm{~d}_{3}$ and $\mathrm{d}_{4}$, respectively. The sequence of distances between each AP and MS can be expressed by:

$$
\mathrm{d}_{1}<\mathrm{d}_{2}<\mathrm{d}_{3}<\mathrm{d}_{4}
$$

And, for the received signal strength sequence from each AP it is shown that:

$$
\text { RSSI1 > RSSI }>\text { RSSI } 3>\text { RSSI4 }
$$

When APs are close together or located on a line, the distribution of APs is said to be weak and the DOP value is high. As shown in Fig. 3, AP1, AP2 and AP3 are located on a line (Case A), the corresponding DOP value is high. However, the DOP value of Case B is low because the APs (AP1, AP2 and AP4) are far from each other. In this paper, DOP3 represents DOP value of the AP combination AP1, AP2 and AP3. Also, DOP4 represents DOP value of AP combination AP1, AP2 and AP4.

For simplicity, we assume that the AP1 and AP2 are dominant APs which provide very strong signals compared with those of the other APs. Then we just make selection

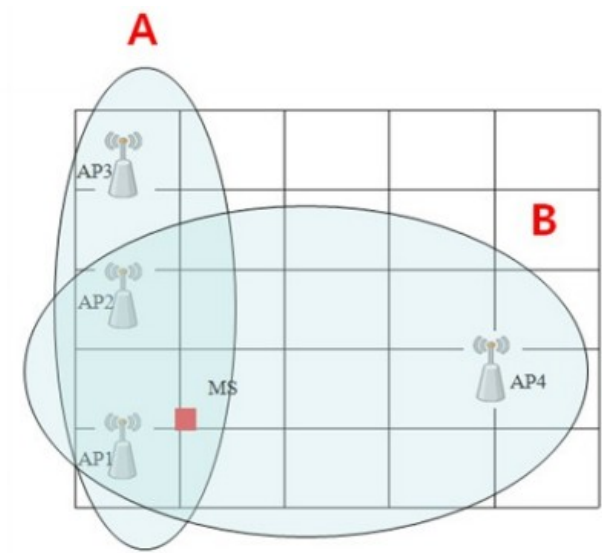

Fig. 4. Configuration of APs between AP3 and AP4 by considering their corresponding DOPs, respectively. That is, in case that the RSSI of AP3 is a little stronger than that of AP4 but DOP3 is higher than DOP4, we may select the AP set of AP1, AP2 and AP4 for more accurate positioning. So we experimented with how RSSI and DOP affect the positioning error. For our experimental setup, we placed APs in an area of $15 \times 15 \mathrm{~m}^{2}$, in the IT No. 3 building of Kyungpook National University. We executed two experiments to verify the proposed algorithm. Each experiment is divided into three kinds of situations. The first situation is that the DOP3 rating is poor, the second situation is that the DOP3 rating is fair and the third situation is that the DOP3 rating is moderate.

\section{Performance and Analysis Results}

\subsection{Analysis of the first experimental results}

Fig. 5 shows that the configuration of AP4 for all situations. We set the distance from AP1 to MS $2.7 \mathrm{~m}$, from AP2 to MS $3.7 \mathrm{~m}$, from AP3 to MS $4.5 \mathrm{~m}$, from $\mathrm{AP}_{1 \mathrm{n}}$ to MS 5m, from $\mathrm{AP}_{2 \mathrm{n}}$ to MS 5.5m, from $\mathrm{AP}_{3 \mathrm{n}}$ to MS $6 \mathrm{~m}$, and from $\mathrm{AP}_{4 \mathrm{n}}$ to MS 6.5m; $(\mathrm{n}=1,2,3 \ldots 33)$. Angle $\alpha$ is the angle between the AP2 and AP4 as viewed from the MS, Angle $\theta_{\mathrm{j}}$ is the deviation angle from the first AP4 to the last with an increment of $5^{\circ}(\mathrm{j}=1,2,3 \ldots 32)$. In order to ensure that the DOP4 rating is fair, moderate and good, we set the first AP4 at an angle of $60^{\circ}$ with respect to AP2.

From the Fig. 6, we can get the DOP4 rating and the distribution range of AP4 corresponding to each rating.

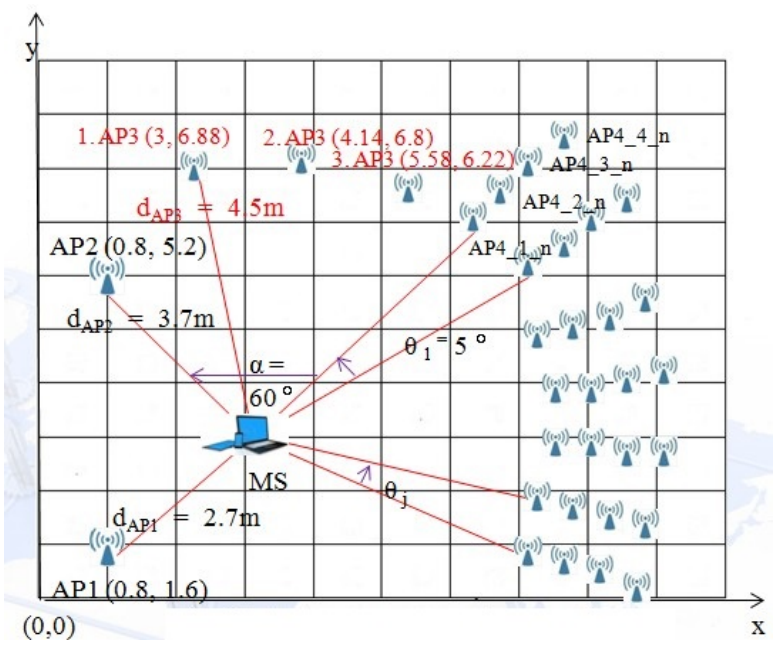

Fig. 5. Configuration of APs of Experiment 1

Table 3. DOP4 rating in experiment 1

\begin{tabular}{c|c}
\hline DOP4 Rating & ${\text { Distribution Range of AP4 }\left(\alpha, \text { unit: }^{\circ}\right)}^{\circ}$ \\
\hline Fair & $(65,90)$ and $(210,225)$ \\
\hline Moderate & $(90,120)$ and $(180,210)$ \\
\hline Good & $(120,180)$ \\
\hline
\end{tabular}




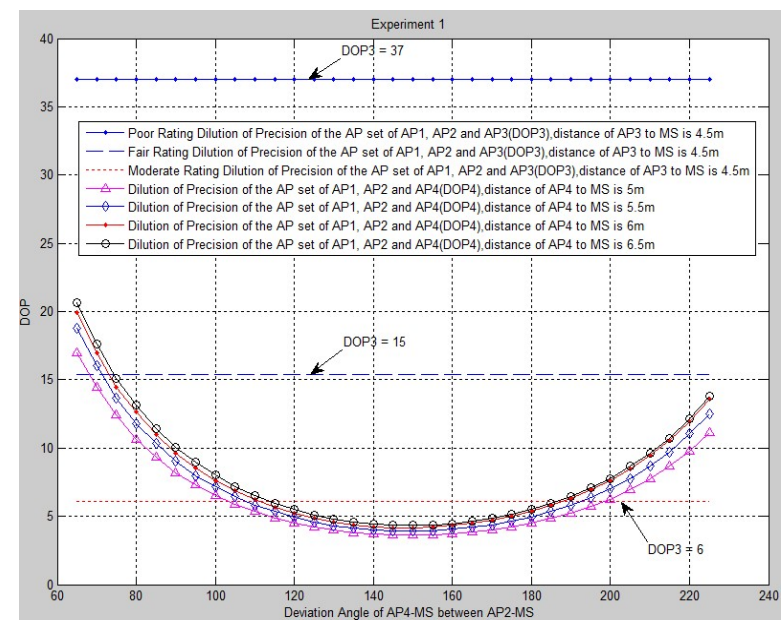

Fig. 6. Comparison of DOP3 and DOP4 in the experiment 1

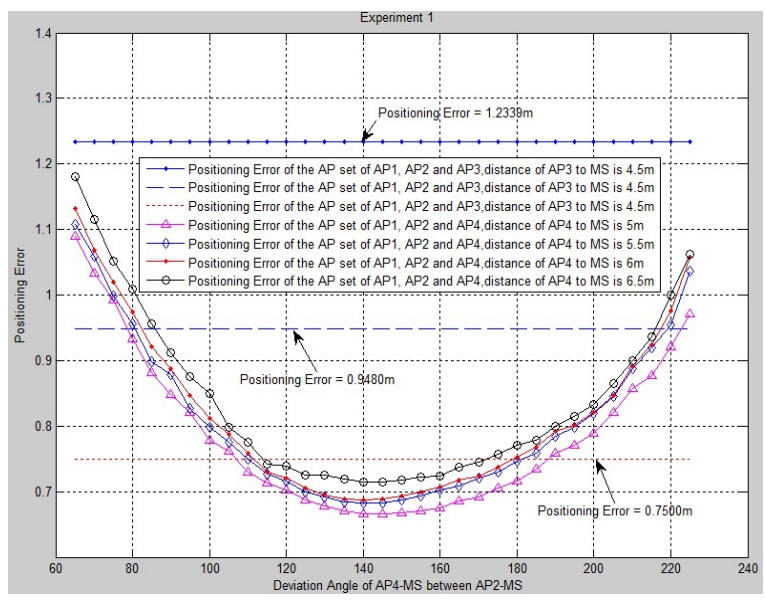

Fig. 7. Positioning error comparison between the AP combination of AP1, AP2 and AP4 and the AP combination of AP1, AP2 and AP3 in experiment 1

In Fig. 6, the order of the first reference to the third reference is from top to bottom. The first reference DOP3 rating is poor, the second reference DOP3 rating is fair and the third DOP3 rating is moderate.

Fig. 7 shows the result of AP selection. If the DOP3 rating is poor, and the DOP4 rating is also at a poor level, then we will select the AP combination AP1, AP2 and AP3 for positioning, If DOP4 belongs to other levels, for example fair, moderate or good level, we will select the AP combination AP1, AP2 and AP4 for positioning. In general, If the DOP4 rating is not better than the DOP3 rating, we only select the AP combination AP1, AP2 and AP3 for positioning, but if the DOP4 rating is better than the DOP3 rating, even when the distance from AP4 to MS is farther than the distance from AP3 to MS, we select the AP combination AP1, AP2 and AP4 for positioning which can obtain a better positioning accuracy than selecting the AP combination AP1, AP2 and AP3. This result was confirmed by experiment 1 . Finally, we can summarize the result as shown in Table 4.
Table 4. Result of APs selection in experiment 1

\begin{tabular}{|c|c|c|c|c|c|}
\hline \multirow[t]{2}{*}{ D.D. } & \multirow{2}{*}{$\begin{array}{l}\text { DOP3 } \\
\text { Rating }\end{array}$} & \multirow{2}{*}{$\begin{array}{l}\text { DOP4 } \\
\text { Rating }\end{array}$} & \multirow{2}{*}{$\begin{array}{c}\text { Distribution } \\
\text { of AP4 } \\
\left(\alpha, \text { unit: }^{\circ}\right)\end{array}$} & \multicolumn{2}{|c|}{$\begin{array}{l}\text { APs Selection } \\
\text { (AP1, AP2, APx) }\end{array}$} \\
\hline & & & & $\mathrm{AP} 3$ & $\mathrm{AP} 4$ \\
\hline \multirow{5}{*}{$0.5 \mathrm{~m}$} & \multirow{4}{*}{ Poor } & Poor & $(0,65) \&(225, \sim)$ & [ & \\
\hline & & Fair & $(65,90) \&(210,225)$ & & - \\
\hline & & Moderate & $(90,120) \&(180,210)$ & & - \\
\hline & & Good & $(120,180)$ & & [ \\
\hline & \multirow{3}{*}{ Fair } & Fair & $(65,90) \&(210,225)$ & [ & \\
\hline \multirow{4}{*}{$2 \mathrm{~m}$} & & Moderate & $(90,120) \&(180,210)$ & & घ \\
\hline & & Good & $(120,180)$ & & [ \\
\hline & \multirow{2}{*}{ Moderate } & Moderate & $(90,120) \&(180,210)$ & घ & \\
\hline & & Good & $(120,180)$ & & [ \\
\hline
\end{tabular}

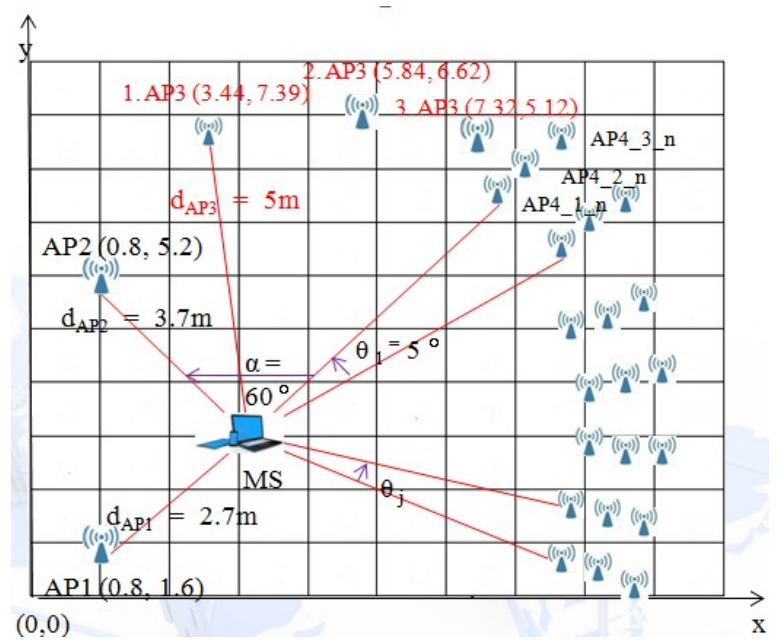

Fig. 8. Configuration of APs of Experiment 2

The symbol " $\square$ " means the selected AP; APx: Represents AP3 or AP4; D.D.: Distance Difference Difference in the distance from MS to AP3, the distance from AP4 to MS is $0.5 \mathrm{~m}, 1 \mathrm{~m}, 1.5 \mathrm{~m}$, and $2 \mathrm{~m}$ farther than AP3 to MS;

\subsection{Analysis of the second experimental results}

Fig. 8 shows the distribution of AP4s in experiment 2 is the same as experiment 1 , the only difference is that the distance from AP3 to MS was set to $5 \mathrm{~m}$. The distance from AP4_1_n to MS is set to $5.5 \mathrm{~m}$, the distance from AP4_2_n is set to $6 \mathrm{~m}$ and the distance from AP4 3 _n is set to $6.5 \mathrm{~m}$. $(\mathrm{n}=1,2,3 \ldots 33)$.

Because the configuration of AP4s is the same as experiment 1, we know that the DOP4 rating and the distribution range of AP4 corresponding to each rating is the same as that of experiment 1 , given in Table 3 .

Fig. 9 shows the result of AP selection. We can summarize the results as shown in Table 5.

The symbol " $\square$ " means the selected AP; APx: Represents AP3 or AP4; D.D.: Distance Difference - Difference in the distance from MS to AP3, the distance from AP4 to MS is $0.5 \mathrm{~m}, 1 \mathrm{~m}$, and $1.5 \mathrm{~m}$ farther than AP3 to MS.

We propose a selection criteria of AP, $f_{i}$, as follows: 
Table 5. Result of APs selection in experiment 2

\begin{tabular}{|c|c|c|c|c|c|}
\hline \multirow[t]{2}{*}{ D.D. } & \multirow[t]{2}{*}{$\begin{array}{l}\text { DOP3 } \\
\text { Rating }\end{array}$} & \multirow[t]{2}{*}{$\begin{array}{l}\text { DOP4 } \\
\text { Rating }\end{array}$} & \multirow[t]{2}{*}{$\begin{array}{c}\text { Distribution } \\
\text { of AP4 } \\
\left(\alpha, \text { unit: }^{\circ}\right)\end{array}$} & \multicolumn{2}{|c|}{$\begin{array}{c}\text { APs } \\
\text { Selection } \\
\text { (AP1, AP2, } \\
\text { APx) }\end{array}$} \\
\hline & & & & AP3 & AP4 \\
\hline \multirow{9}{*}{$1.5 \mathrm{~m}$} & \multirow{4}{*}{ Poor } & Poor & $(0,65) \&(225, \sim)$ & $\mathbf{\square}$ & \\
\hline & & Fair & $(65,90) \&(210,225)$ & & $\mathbf{\square}$ \\
\hline & & Moderate & $(90,120) \&(180,210)$ & & $\mathbf{\square}$ \\
\hline & & Good & $(120,180)$ & & $\mathbf{\square}$ \\
\hline & \multirow{3}{*}{ Fair } & Fair & $(65,90) \&(210,225)$ & $\mathbf{\square}$ & \\
\hline & & Moderate & $(90,120) \&(180,210)$ & & $\mathbf{\square}$ \\
\hline & & Good & $(120,180)$ & & $\mathbf{\square}$ \\
\hline & \multirow{2}{*}{ Moderate } & Moderate & $(90,120) \&(180,210)$ & $\mathbf{\square}$ & \\
\hline & & Good & $(120,180)$ & & $\mathbf{\square}$ \\
\hline
\end{tabular}

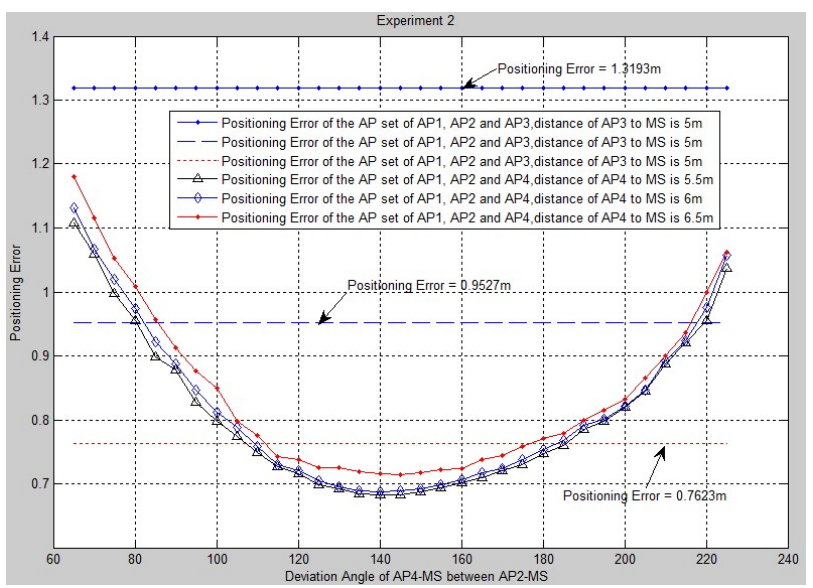

Fig. 9. Positioning error comparison between the AP combination of AP1, AP2 and AP4 and the AP combination of AP1, AP2 and AP3 in experiment 2

First part and second one reflect RSSI information and DOP information for AP selection, respectively. The lower value of $f_{i}$ guarantees the more precise positioning.

$$
f_{i}=\left(\left|R S S I_{1}+R S S I_{2}+R S S I_{i}\right| \times \sqrt{v a r}\right) \times\left(1+\ln D O P_{i}\right)
$$

Proposed selection procedure is as follows:

Step 1: Calculate DOP3 and DOP4, respectively

Step 2: Calculate the $f_{3}$ and $f_{4}$, respectively, in Eq. (9)

Step 3: Compare $f_{3}$ and $f_{4}$, select AP more lower factor.

\subsection{Performance analytic results}

From the above experiments we can say that the proposed method using IEEE 802.11 RSSI measurements and AP configuration information could reduce more the average positioning error and the maximum positioning error than only using the RSSI attenuation model.

Table 6 shows that in case that the distance from AP3 to MS is $4.5 \mathrm{~m}$, the reduction rate of average and maximum positioning error, by the AP combination AP1, AP2 and
Table 6. Error reduction results of experiment 1

\begin{tabular}{|c|c|c|c|c|c|c|}
\hline $\begin{array}{l}\text { DOP3 } \\
\text { Rating }\end{array}$ & $\begin{array}{l}\text { Positiong } \\
\text { Error(m) }\end{array}$ & $\begin{array}{l}\text { DOP4 } \\
\text { Rating }\end{array}$ & $\begin{array}{c}\text { Maximum } \\
\text { Error(m) }\end{array}$ & $\begin{array}{l}\text { Average } \\
\text { Error }(\mathrm{m})\end{array}$ & $\begin{array}{c}\mathrm{ME} \\
\text { reduction }\end{array}$ & $\begin{array}{c}\mathrm{AE} \\
\text { reduction }\end{array}$ \\
\hline \multirow{3}{*}{ Poor } & \multirow{3}{*}{1.2339} & Fair & 1.1805 & 1.0192 & $4.33 \%$ & $17.4 \%$ \\
\hline & & Moderate & 0.9119 & 0.7936 & $26.1 \%$ & $35.68 \%$ \\
\hline & & Good & 0.7259 & 0.7053 & $41.17 \%$ & $42.84 \%$ \\
\hline \multirow{2}{*}{ Fair } & \multirow{2}{*}{0.9480} & Moderate & 0.9119 & 0.7936 & $3.81 \%$ & $16.29 \%$ \\
\hline & & Good & 0.7259 & 0.7053 & $23.43 \%$ & $25.6 \%$ \\
\hline Moderate & 0.7500 & Good & 0.7259 & 0.7053 & $3.21 \%$ & $5.96 \%$ \\
\hline
\end{tabular}

Table 7. Error reduction results of experiment 2

\begin{tabular}{c|c|c|c|c|c|c}
\hline $\begin{array}{c}\text { DOP3 } \\
\text { Rating }\end{array}$ & $\begin{array}{c}\text { Positiong } \\
\text { Error(m) }\end{array}$ & $\begin{array}{c}\text { DOP4 } \\
\text { Rating }\end{array}$ & $\begin{array}{c}\text { Maximum } \\
\text { Error }(\mathrm{m})\end{array}$ & $\begin{array}{c}\text { Average } \\
\text { Error(m) }\end{array}$ & $\begin{array}{c}\text { ME } \\
\text { reduction }\end{array}$ & $\begin{array}{c}\text { AE } \\
\text { reduction }\end{array}$ \\
\hline \multirow{3}{*}{ Poor } & 1.3793 & Fair & 1.1805 & 1.0192 & $10.52 \%$ & $22.75 \%$ \\
\cline { 3 - 7 } & & Moderate & 0.9119 & 0.7936 & $30.88 \%$ & $39.85 \%$ \\
\cline { 2 - 7 } & Good & 0.7259 & 0.7053 & $44.98 \%$ & $46.54 \%$ \\
\hline \multirow{2}{*}{ Fair } & 0.9527 & Moderate & 0.9119 & 0.7936 & $4.28 \%$ & $16.7 \%$ \\
\cline { 2 - 7 } & Good & 0.7259 & 0.7053 & $23.81 \%$ & $25.97 \%$ \\
\hline Moderate & 0.7623 & Good & 0.7259 & 0.7053 & $4.78 \%$ & $7.48 \%$ \\
\hline $\begin{array}{l}\text { ME: Maximum Error; AE: Average Error; Positioning Error(of locati } \\
\text { on using the AP combination AP1, AP2 and AP 4); Average E } \\
\text { rror(of location using the AP combination AP1, AP2 and AP3) }\end{array}$
\end{tabular}

AP4, are $23.96 \%$ and $17 \%$, respectively. Table 7 shows that in case that the distance from AP3 to MS is $5 \mathrm{~m}$, the average reduction rate of average and maximum positioning error, deduced by the AP combination AP1, AP2 and AP4, are $26.61 \%$ and $19.83 \%$, respectively.

\section{Conclusion}

As the wireless network technology is developing faster and faster, many buildings are covered with wireless networks, but the access points are set in a concentrated and complex arrangement. These concentrated and complex AP sets lead to positioning errors occuring in indoor environments. To overcome this problem, in this paper, we have presented an indoor positioning method considering the AP combination geometric distribution adopting dilution of precision (DOP) and using IEEE 802.11 RSSI measurements. The experimental results show that the proposed method can reduce the average positioning error around $25 \%$, and it is always better than the other existing method [11] which only uses IEEE 802.11 measurements without adopting AP configuration information.

\section{Acknowledgements}

This research was supported by Kyungpook National University Research Fund, 2013 and has been supported by the national GNSS Research Center program of Defense Acquisition Program Administration and Agency for Defense Development. 


\section{References}

[1] I. Getting. "The Global Positioning System," IEEE Spectrum. 30(12): 36-47. December 1993.

[2] http://www.ubisense.net,

[3] Liu, Hui, et al. "Survey of wireless indoor positioning techniques and systems." Systems, Man, and Cybernetics, Part C: Applications and Reviews, IEEE Transactions on 37.6 (2007): 1067-1080.

[4] A. D. Angelis, J. O. Nilsson, I. Skog, P. Handel and P. Carbone, "Indoor Positioning By Ultra Wide Band Radio Aided Inertial Navigation," XIX IMEKO World Congress Fundamental and Applied Metrology, pp. 574-579, September 6-11, 2009, Lisbon, Portugal.

[5] http://en.wikipedia.org/wiki/Wi-Fi, Jun. 2013.

[6] IEEE standard for Information Technology, Telecommunications and Information Exchange between System, Local and Metropolitan Area Network. Part II: Wireless LAN Medium Access Control (MAC) and Physical Layer (PHY) Specifications, IEEE standard 802.11,1999.

[7] E. D. Kaplan, C. J. Hegarty, "Understanding GPS principles and applications," second edition

[8] http://en.wikipedia.org/wiki/Dilution_of_precision_ (GPS), Jun. 2013.

[9] S. Park, D. Park, A. S. Kim, J. Park, S. Kim, C. Lim, and J. G. Park, "A study on enhanced indoor localization method through IEEE 802.11 signal strength measurements," KSII ICONI pp. 761-765, December 2010.

[10] Hyun-Hun Cho, Rak-Hee Lee, Joon-Goo Park, "Adaptive Parameter Estimation Method for Wireless Localization Using RSSI Measurements," Journal of Electrical Engineering \& Technology, Vol. 6, No. 6, pp. 883-887, 2011.

[11] Hasani, M, "Hybrid WLAN-RFID Indoor Localization Solution Utilizing Textile Tag," IEEE Antennas and Wireless Propagation Letters, Vol. 14, pp. 13581361, 2015.

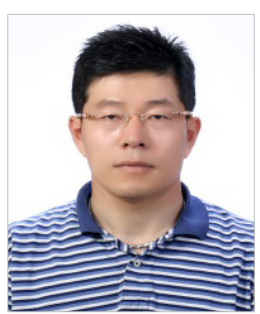

Joon Goo Park He received Ph.D. degree from Seoul National University in 2001. He is a professor at the School of Electronics Engineering at Kyungpook National University. Research interests are GPS, mobile software navigation algorithm. 